\title{
Las enfermedades periodontales en pacientes esquizofrénicos. Un estudio de casos-controles
}

\author{
VELASCO ORTEGA E* \\ MONSALVE GUIL L** \\ CASAS BARQUERO $\mathbf{N} * * *$ \\ VELASCO PONFERRADA C** \\ MEDEL SOTERAS R****
}

\begin{abstract}
Velasco Ortega E, Monsalve Guil L, Casas Barquero N, Velasco Ponferrada C, Medel Soteras R. Las enfermedades periodontales en pacientes esquizofrénicos. Un estudio de casos-controles. Av Periodon Implantol. 2005; 17, 1: 235-243.
\end{abstract}

\section{RESUMEN}

El objetivo del presente trabajo era valorar las condiciones periodontales en pacientes esquizofrénicos comparados con pacientes control.

Pacientes y Métodos. El estudio se realizó en 50 pacientes esquizofrénicos ingresados en la Unidad de Psiquiatría del Hospital Universitario Virgen Macarena de Sevilla y se compararon con 50 pacientes adultos de los que acuden a recepción de la Facultad de Odontología de la Universidad de Sevilla, excluyendo de este último grupo aquellos pacientes que presentaban alguna patología sistémica en el momento de la exploración o en los seis meses previos a ésta. En ambos grupos se valoró el estado periodontal según el protocolo de la OMS (CPITN).

Resultados y Discusión. Los pacientes esquizofrénicos presentaron una significativa mayor extensión de sextantes excluídos $(1,64$ vs 0,42$)$ y una mayor prevalencia de bolsas moderadas $(25,6 \%$ vs $22,4 \%)$ mientras que los pacientes controles presentaron una mayor prevalencia de cálculo $(69,4 \%$ vs $60,5 \%)$ y bolsas profundas $(6,1 \%$ vs $4,7 \%)$. Las condiciones periodontales presentaban una tendencia ascendente con la edad. Los hombres (esquizofrénicos y controles) $(32,4 \%$ y $31,3 \%$, respectivamente) tenían mayor prevalencia de bolsas periodontales que las mujeres (22,2\% and $23,5 \%)$. Además este estudio muestra una mayor prevalencia y extension de las bolsas periodontales entre los pacientes fumadores (esquizofrénicos y controles).

Conclusiones. La relación entre los problemas periodontales y mentales debe constituir un campo de investigación epidemiológica y clinica para el conocimiento de la historia natural de las enfermedades periodontales .

\section{PALABRAS CLAVE}

Condiciones periodontales, pacientes esquizofrénicos, CPIT'N, trastornos mentales, enfermedad periodontal.

Aceptado para publicación: Enero 2005

*** Profesora Asociada de Psicología y Psiquiatría. Facultad de Odontología de Sevilla. Médico Adjunto del Servicio de Psiquiatría del Hospital Universitario Virgen Macarena de Sevilla.

**** Profesor Asociado de Odontología Integrada de Adultos. Facultad de Odontología. Universidad de Sevilla.
} 


\section{INTRODUCCIÓN}

Las enfermedades periodontales constituyen algunas de las condiciones orales más frecuentes de la humanidad. Globalmente, la gingivitis, la presencia de cálculo y los estadíos iniciales de la enfermedad periodontal son muy prevalentes en la población adulta. La periodontitis avanzada, aunque menos prevalente puede causar la pérdida de dientes en un $5-15 \%$ de la población general (1).

En los paises occidentales, se ha demostrado que el tabaco es uno de los factores de riesgo más importantes para las enfermedades periodontales de los adultos, y que su consumo es responsable de más de la mitad de los casos de periodontitis en este grupo etario (2).

Los pacientes que sufren enfermedades mentales crónicas representan un grupo de riesgo para la salud oral (3). Se ha demostrado que los enfermos psiquiátricos presentan una mayor frecuencia y severidad de caries, enfermedades periodontales y lesiones de la mucosa oral (4-6). Así mismo, se ha investigado la importancia de los factores psicosociales en la etiopatogenia y en la respuesta al tratamiento de las enfermedades periodontales (7-8).

Aunque los estudios periodontales son numerosos, sin embargo, no existen investigaciones que comparen específicamente los hallazgos clínicos periodontales entre pacientes con y sin trastornos mentales, por lo que el objetivo del presente estudio era valorar la prevalencia y extensión de las condiciones periodontales en pacientes con esquizofrenia y compararla con pacientes controles sanos.

\section{PACIENTES Y MÉTODOS}

Un estudio comparativo de las condiciones periodontales fué realizado en dos grupos de pacientes, esquizofrénicos y sanos controles que fueron elegidos de forma aleatoria, y estratificados según la edad y el sexo.

El examen periodontal se realizó entre los pacientes esquizofrénicos que habían sido ingresados en la Unidad de Agudos de Psiquiatría del Hospital Universitario Virgen Macarena, adjunto a la Facultad de Medicina de la Universidad de Sevilla. Los pacientes sanos controles fueron elegidos entre los pacientes adultos que acudían a recepción para tratamiento odontológico en la Facultad de Odontología de la Universidad de Sevilla. Fueron excluidos para este estudio como pacientes control, aquellos sujetos que presentaban cualquier enfermedad sistémica y/o consumían cualquier tratamiento farmacológico en el momento de la exploración, o habían padecido patología médica aguda los seis meses antes del examen.

El examen periodontal fué realizado en la Facultad de Odontología de Sevilla para los pacientes controles sanos y en la Unidad de Psiquiatría del Hospital Universitario Virgen Macarena de Sevilla para los pacientes esquizofrénicos. El examen periodontal se realizó utilizando el Indice Periodontal Comunitario de Necesidades de Tratamiento (en inglés, CPITN) siguiendo las recomendaciones del Manual de Investigación Oral de la Organización Mundial de la Salud (9).

Los datos sobre las condiciones periodontales fueron evaluados en ambos grupos de pacientes esquizofrénicos y controles sanos, con respecto a las variables demográficas y las variables clínicas. Las diferencias estadísticas fueron identificadas según el test de la chi-cuadrado (variables cualitativas) y de la varianza (variables cuantitativas), aceptándose como estadísticamente significativa, cuando $\mathrm{p}<0,05$.

\section{RESULTADOS}

\section{PACIENTES}

Se estudiaron 50 pacientes esquizofrénicos (39 varones y 11 mujeres) con una edad media de 39,9 años (rango: 17-64 años) y 50 pacientes sanos control (33 varones y 17 mujeres) con una edad media de 39,5 años (rango: 20-67 años). No existían diferencias significativas al comparar ambos grupos de pacientes, con respecto a la edad (ANOVA; $p=0,8542$ ) y el sexo (chi-cuadrado; $\mathrm{p}=0,18145$ ).

Entre los pacientes esquizofrénicos, 29 eran fumadores (58\%); mientras que entre los pacientes control, 26 fumaban $(52 \%)$. Estas diferencias no eran significativas (chi-cuadrado, $p=, 012000$ ).

\section{ESTADO PERIODONTAL}

\section{Prevalencia de las condiciones periodontales}

Los pacientes esquizofrénicos presentaban un mayor porcentaje de gingivitis $(4,7 \%)$ y bolsas periodontales moderadas (25,6\%); mientras que los pacientes sanos control presentaban una mayor frecuencia de cálculo $(69,4 \%)$ y bolsas periodontales profundas $(6,1 \%)$ (Tabla 1). Estas diferencias no eran estadísticamente significativas (chi-cuadrado; $p=0,52132$ ).

En los pacientes esquizofrénicos se incrementaban las bolsas moderadas y profundas con la edad desde 


\begin{tabular}{|lccccccc|}
\hline \multicolumn{7}{|c|}{ TABLA } & 1.- DISTRIBUCIÓN DE LOS PACIENTES SEGÚN LA PREVALENCIA (\%) \\
DE LAS CONDICIONES PERIODONTALES & & \\
\hline CPITN & $\mathbf{N}^{\circ}$ Examinados & $\mathbf{N}^{\circ}$ dentados & $\mathbf{0}$ & $\mathbf{1}$ & $\mathbf{2}$ & $\mathbf{3}$ & $\mathbf{4}$ \\
\hline Esquizofrénicos & 50 & 43 & $4,7 \%$ & $4,7 \%$ & $60,5 \%$ & $25,6 \%$ & $4,7 \%$ \\
Controles & 50 & 49 & $0 \%$ & $2 \%$ & $69,4 \%$ & $22,4 \%$ & $6,1 \%$ \\
\hline
\end{tabular}

\begin{tabular}{|c|c|c|c|c|c|c|c|}
\hline \multicolumn{8}{|c|}{$\begin{array}{l}\text { TABLA 2.- LA PREVALENCIA (\%) DE LAS CONDICIONES PERIODONTALES } \\
\text { SEGÚN LA EDAD DE LOS PACIENTES }\end{array}$} \\
\hline CPITN & $\mathbf{N}^{\circ}$ Examinados & $\mathbf{N}^{\circ}$ dentados & 0 & 1 & 2 & 3 & 4 \\
\hline \multicolumn{8}{|c|}{ Esquizofrénicos } \\
\hline$<35$ años & 13 & 13 & $7,7 \%$ & $15,4 \%$ & $69,2 \%$ & $7,7 \%$ & $0 \%$ \\
\hline 36-45 años & 25 & 24 & $4,2 \%$ & $0 \%$ & $62,5 \%$ & $29,2 \%$ & $4,2 \%$ \\
\hline$>46$ años & 12 & 6 & $0 \%$ & $0 \%$ & $33,3 \%$ & $50 \%$ & $16,7 \%$ \\
\hline Total & 50 & 43 & $4,7 \%$ & $4,7 \%$ & $60,5 \%$ & $25,6 \%$ & $4,7 \%$ \\
\hline \multicolumn{8}{|l|}{ Controles } \\
\hline$<35$ años & 19 & 19 & $0 \%$ & $0 \%$ & $73,7 \%$ & $21,1 \%$ & $5,3 \%$ \\
\hline 36-45 años & 17 & 17 & $0 \%$ & $0 \%$ & $70,6 \%$ & $23,5 \%$ & $5,9 \%$ \\
\hline$>46$ años & 14 & 13 & $0 \%$ & $1,7 \%$ & $61,5 \%$ & $23,1 \%$ & $7,7 \%$ \\
\hline Total & 50 & 49 & $0 \%$ & $2 \%$ & $69,4 \%$ & $22,4 \%$ & $6,1 \%$ \\
\hline
\end{tabular}

\begin{tabular}{|c|c|c|c|c|c|c|c|}
\hline \multicolumn{8}{|c|}{$\begin{array}{l}\text { TABLA 3.- LA PREVALENCIA (\%) DE LAS CONDICIONES PERIODONTALES } \\
\text { SEGÚN EL SEXO DE LOS PACIENTES }\end{array}$} \\
\hline CPITN & $\mathbf{N}^{\circ}$ Examinados & $\mathbf{N}^{\circ}$ dentados & 0 & 1 & 2 & 3 & 4 \\
\hline \multicolumn{8}{|c|}{ Esquizofrénicos } \\
\hline Hombres & 39 & 34 & $5,9 \%$ & $5,9 \%$ & $55,9 \%$ & $26,5 \%$ & $5,9 \%$ \\
\hline Mujeres & 11 & 9 & $0 \%$ & $0 \%$ & $77,8 \%$ & $22,2 \%$ & $0 \%$ \\
\hline Total & 50 & 43 & $4,7 \%$ & $4,7 \%$ & $60,5 \%$ & $25,6 \%$ & $4,7 \%$ \\
\hline \multicolumn{8}{|l|}{ Controles } \\
\hline Hombres & 33 & 32 & $0 \%$ & $3,1 \%$ & $65,6 \%$ & $21,9 \%$ & $9,4 \%$ \\
\hline Mujeres & 17 & 17 & $0 \%$ & $0 \%$ & $76,5 \%$ & $23,5 \%$ & $0 \%$ \\
\hline Total & 50 & 49 & $0 \%$ & $2 \%$ & $69,4 \%$ & $22,4 \%$ & $6,1 \%$ \\
\hline
\end{tabular}

el $7,7 \%$ en los menores de 35 años hasta el $66,7 \%$ en los mayores de 46 años. Estas diferencias no eran estadísticamente significativas (chi-cuadrado; $\mathrm{p}=$ 0,16502). Esta tendencia era menor en los pacientes control ya que solamente existía un incremento moderado de la prevalencia de bolsas profundas desde el $5,3 \%$ en los pacientes más jóvenes hasta el 7,7\% entre los pacientes de más edad (Tabla 2). Estas diferencias no eran estadísticamente significativas (chi-cuadrado; $\mathrm{p}=0,80340$ ).

Con respecto al sexo, tanto los pacientes esquizofrénicos como los pacientes sanos control varones $(32,4 \%$ y $31,3 \%$ ) presentaban mayor destrucción periodontal (prevalencia de bolsas periodontales) que las mujeres $(22,2 \%$ y $23,5 \%)$; aunque estas diferencias no eran estadísticamente significativas (chi-cuadrado; $\mathrm{p}=$ 0,80198 y $p=0,57897$; respectivamente) (Tabla 3 ).

Los pacientes fumadores, esquizofrénicos y sanos control, presentaban una mayor prevalencia de bolsas periodontales $(38,5 \%$ y $32 \%)$, respectivamente, que los pacientes no fumadores $(17,6 \%$ y $25 \%)$. Estas diferencias no eran estadísticamente significativas (chicuadrado; $p=0,08694$ y $p=0,67718$; respectivamente) (Tabla 4). 


\begin{tabular}{|c|c|c|c|c|c|c|c|}
\hline \multicolumn{8}{|c|}{$\begin{array}{c}\text { TABLA 4.- LA PREVALENCIA (\%) DE LAS CONDICIONES PERIODONTALES } \\
\text { SEGÚN EL HÁBITO DE FUMAR DE LOS PACIENTES }\end{array}$} \\
\hline CPITN & $\mathbf{N}^{\circ}$ Examinados & $\mathbf{N}^{\circ}$ dentados & 0 & 1 & 2 & 3 & 4 \\
\hline \multicolumn{8}{|c|}{ Esquizofrénicos } \\
\hline Fumadores & 29 & 26 & $0 \%$ & $0 \%$ & $61,5 \%$ & $30,8 \%$ & $7,7 \%$ \\
\hline No fumadores & 21 & 17 & $11,8 \%$ & $11,8 \%$ & $58,8 \%$ & $17,6 \%$ & $0 \%$ \\
\hline Total & 50 & 43 & $4,7 \%$ & $4,7 \%$ & $60,5 \%$ & $25,6 \%$ & $4,7 \%$ \\
\hline \multicolumn{8}{|l|}{ Controles } \\
\hline Hombres & 26 & 25 & $0 \%$ & $4 \%$ & $64 \%$ & $24 \%$ & $8 \%$ \\
\hline Mujeres & 24 & 24 & $0 \%$ & $0 \%$ & $75 \%$ & $20,8 \%$ & $4,2 \%$ \\
\hline Total & 50 & 49 & $0 \%$ & $2 \%$ & $69,4 \%$ & $22,4 \%$ & $6,1 \%$ \\
\hline
\end{tabular}

\begin{tabular}{|c|c|c|c|c|c|c|c|c|}
\hline \multicolumn{9}{|c|}{$\begin{array}{l}\text { TABLA 5.- DISTRIBUCIÓN DE LOS PACIENTES SEGÚN LA EXTENSIÓN } \\
\text { (N' MEDIO DE SEXTANTES) DE LAS CONDICIONES PERIODONTALES }\end{array}$} \\
\hline CPITN & $\mathbf{N}^{\circ}$ Examinados & $\mathbf{N}^{\circ}$ dentados & 0 & 1 & 2 & 3 & 4 & $\mathbf{x}$ \\
\hline Esquizofrénicos & 50 & 43 & 0,70 & 0,30 & 2,88 & 0,97 & 0,04 & 1,64 \\
\hline Controles & 50 & 49 & 0,74 & 0,63 & 3,24 & 0,85 & 0,12 & 0,42 \\
\hline
\end{tabular}

\begin{tabular}{|c|c|c|c|c|c|c|c|c|}
\hline \multicolumn{9}{|c|}{$\begin{array}{c}\text { TABLA 6.- LA EXTENSIÓN (N MEDIO DE SEXTANTES) DE LAS CONDICIONES } \\
\text { PERIODONTALES SEGÚN LA EDAD DE LOS PACIENTES }\end{array}$} \\
\hline CPITN & $\mathbf{N}^{\circ}$ Examinados & $\mathbf{N}^{\circ}$ dentados & 0 & 1 & 2 & 3 & 4 & $\mathbf{x}$ \\
\hline \multicolumn{9}{|c|}{ Esquizofrénicos } \\
\hline$<35$ años & 13 & 13 & 1,92 & 1,07 & 2,92 & 0,07 & 0 & 0 \\
\hline 36-45 años & 25 & 24 & 0,40 & 0,04 & 3,44 & 0,60 & 0,04 & 1,48 \\
\hline > 46 años & 12 & 6 & 0 & 0 & 1,66 & 0,50 & 0,08 & 3,75 \\
\hline Total & 50 & 43 & 0,70 & 0,30 & 2,88 & 0,97 & 0,04 & 1,64 \\
\hline \multicolumn{9}{|l|}{ Controles } \\
\hline$<35$ años & 19 & 19 & 0,84 & 0,96 & 3,63 & 0,78 & 0,10 & 0,10 \\
\hline 36-45 años & 17 & 17 & 0,70 & 1,21 & 3,17 & 0,70 & 0,17 & 0,52 \\
\hline$>46$ años & 14 & 13 & 0,53 & 1,31 & 2,76 & 1,15 & 0,07 & 0,76 \\
\hline Total & 50 & 49 & 0,74 & 0,63 & 3,24 & 0,85 & 0,12 & 0,42 \\
\hline
\end{tabular}

\section{Extensión de las condiciones periodontales}

Los pacientes esquizofrénicos presentaban una mayor extensión en sextantes excluídos y bolsas periodontales moderadas; mientras que los pacientes sanos control presentaban una mayor extensión de cálculo y bolsas periodontales profundas (Tabla 5). Estas diferencias no eran estadísticamente significativas (ANOVA; $\mathrm{p}=0,7559 ; \mathrm{p}=0,2635 ; \mathrm{p}=0,8075 ; \mathrm{p}=$ $0,2220 ; p=0,3785)$ excepto para los sextantes excluí$\operatorname{dos}($ ANOVA; $p=0,0395)$.

Con respecto a la edad, en los pacientes esquizofréni- cos se incrementaba la extensión de los sextantes excluídos con la edad desde una media de 0 sextantes en los menores de 35 años hasta el 3,75 sextantes en los mayores de 46 años. Estas diferencias eran estadísticamente significativas con respecto a la salud periodontal, gingivitis y sextantes excluídos (ANOVA; $p=0,0050 ; p=0,0216 ; p=0,0000$ ) y no eran significativas con respecto al cálculo, bolsas moderadas y bolsas profundas (ANOVA; $p=0,0772 ; p=0,2869 ; p=$ $0,5848)$. Esta tendencia era menor en los pacientes control, con una media de 0,10 sextantes excluidos entree los pacientes más jóvenes hasta los 0,76 sextantes en los más mayores (Tabla 6). Estas diferencias 


\begin{tabular}{|c|c|c|c|c|c|c|c|c|}
\hline \multicolumn{9}{|c|}{$\begin{array}{c}\text { TABLA 7.- LA EXTENSIÓN (N MEDIO DE SEXTANTES) DE LAS CONDICIONES } \\
\text { PERIODONTALES SEGÚN EL SEXO DE LOS PACIENTES }\end{array}$} \\
\hline CPITN & $\mathbf{N}^{\circ}$ Examinados & $\mathbf{N}^{\circ}$ dentados & 0 & 1 & 2 & 3 & 4 & $\mathbf{x}$ \\
\hline \multicolumn{9}{|c|}{ Esquizofrénicos } \\
\hline Hombres & 39 & 34 & 0,71 & 0,38 & 2,69 & 0,91 & 0,05 & 1,71 \\
\hline Mujeres & 11 & 9 & 0,63 & 0 & 3,54 & 1,21 & 0 & 1,36 \\
\hline Total & 50 & 43 & 0,70 & 0,30 & 2,88 & 0,97 & 0,04 & 1,64 \\
\hline \multicolumn{9}{|l|}{ Controles } \\
\hline Hombres & 33 & 32 & 0,68 & 0,68 & 3,03 & 1,06 & 0,18 & 0,34 \\
\hline Mujeres & 17 & 17 & 0,76 & 0,52 & 3,64 & 0,47 & 0 & 0,58 \\
\hline Total & 50 & 49 & 0,74 & 0,63 & 3,24 & 0,85 & 0,12 & 0,42 \\
\hline
\end{tabular}

\begin{tabular}{|c|c|c|c|c|c|c|c|c|}
\hline \multicolumn{9}{|c|}{$\begin{array}{l}\text { TABLA 8.- LA EXTENSIÓN (N MEDIO DE SEXTANTES) DE LAS CONDICIONES } \\
\text { PERIODONTALES SEGÚN EL HÁBITO DE FUMAR DE LOS PACIENTES }\end{array}$} \\
\hline CPITN & $\mathbf{N}^{\circ}$ Examinados & $\mathbf{N}^{\circ}$ dentados & 0 & 1 & 2 & 3 & 4 & $\mathbf{x}$ \\
\hline \multicolumn{9}{|c|}{ Esquizofrénicos } \\
\hline Fumadores & 29 & 26 & 0,27 & 0,03 & 2,19 & 0,55 & 0,06 & 1,68 \\
\hline No fumadores & 21 & 17 & 1,28 & 0,66 & 3,37 & 0,28 & 0 & 1,57 \\
\hline Total & 50 & 43 & 0,70 & 0,30 & 2,88 & 0,97 & 0,04 & 1,64 \\
\hline \multicolumn{9}{|l|}{ Controles } \\
\hline Fumadores & 26 & 25 & 0,36 & 0,44 & 3,48 & 1,04 & 0,20 & 0,48 \\
\hline No fumadores & 24 & 24 & 1,13 & 0,82 & 2,91 & 0,69 & 0,04 & 0,39 \\
\hline Total & 50 & 49 & 0,74 & 0,63 & 3,24 & 0,85 & 0,12 & 0,42 \\
\hline
\end{tabular}

no eran estadísticamente significativas (ANOVA; $p=$ $0,8095 ; p=0,8760 ; p=0,4921 ; p=0,7276 ; p=0,8668 ;)$ excepto para los sextantes excluídos (ANOVA; $p=$ 0,0498).

Con respecto al sexo, las mujeres esquizofrénicas presentaban una mayor extensión de cálculo y destrucción periodontal (bolsas periodontales) que los varones esquizofrénicos que presentaban mayor extensión de sextantes excluídos; aunque estas diferencias no eran estadísticamente significativas (ANOVA; $\mathrm{p}=0,8879 ; \mathrm{p}=0,3529 ; \mathrm{p}=0,2703 ; \mathrm{p}=$ 0,$9559 ; p=0,4536 ; p=0,6463$ ) (Tabla 7). En sentido contrario, los pacientes control varones presentaban mayor extensión de bolsas periodontales que las mujeres sanas control, aunque estas diferencias no eran estadísticamente significativas (ANOVA; $\mathrm{p}=$ 0,$8425 ; p=0,6463 ; p=0,3113 ; p=0,2118 ; p=0,2386$; $\mathrm{p}=0,3078$ ) (Tabla 7).

Los pacientes esquizofrénicos fumadores presentaban una mayor extensión de bolsas periodontales $(0,61)$, y de sextantes excluidos $(1,68)$ que los esquizofrénicos no fumadores (0,28 y 1,57; respectivamente). Estas diferencias no eran estadísticamente significativas
(ANOVA; $\mathrm{p}=0,0652 ; \mathrm{p}=0,0641 ; \mathrm{p}=0,3449 ; \mathrm{p}=$ $0,2277 ; p=0,8554)$ excepto para los sextantes sanos (ANOVA; $p=0,0333$ ) (Tabla 8). Una tendencia similar presentaban los pacientes control fumadores con una mayor extensión de bolsas periodontales $(1,24)$, y de sextantes excluidos $(0,48)$ que los pacientes control no fumadores (0,73 y 0,39; respectivamente). Estas diferencias no eran estadísticamente significativas (ANOVA; $\mathrm{p}=0,2459 ; \mathrm{p}=0,3343 ; \mathrm{p}=0,4564 ; \mathrm{p}=$ 0,$3127 ; p=0,7043$ ) excepto para los sextantes sanos (ANOVA; $p=0,0364$ ) (Tabla 8).

\section{DISCUSIÓN}

Los enfermos psiquiátricos, especialmente, los esquizofrénicos, constituyen un grupo de riesgo para las enfermedades periodontales. Los factores psíquicos pueden interferir en la etiopatogenia de estos trastornos periodontales, la higiene oral es peor con mayores índices de placa y cálculo, la placa periodontopatógena es más agresiva y adherente como consecuencia de la xerostomía y el consumo de tabaco; además otros factores como la hospitalización psiquiátrica y la 
escasa atención odontológica con cuidados periodontales inciden negativamente sobre la salud periodontal de estos pacientes $(5,7,10,11)$.

La esquizofrenia se asocia con un deterioro en la conducta personal lo que provoca un abandono de la higiene oral. Además, la evolución de la enfermedad esquizofrénica puede influir sobre el deterioro periodontal. De hecho, se ha comprobado una relación significativa entre la gravedad de los síntomas psicopatológicos y los signos negativos (abandono emocional, retraso motor) con el grado de higiene oral $(10,12)$.

Los hallazgos clínicos del presente estudio demuestran que no existían diferencias en la prevalencia y la extensión de las condiciones periodontales entre ambos grupos de pacientes esquizofrénicos y controles, excepto con respecto a los sextantes excluidos que mostraban unas diferencias significativas. Los códigos periodontales más frecuentes y extensos eran la presencia de cálculo y las bolsas periodontales. La distribución de estas condiciones periodontales demuestra que la patología periodontal es muy prevalente y extensa tanto entre los pacientes esquizofrénicos como en los pacientes controles.

Otros estudios han valorado los aspectos orales (incluyendo los periodontales) de pacientes esquizofrénicos (13-14). Un estudio norteamericano ha demostrado que los pacientes esquizofrénicos presentan más nivel de placa, cálculo y una mayor tendencia de pérdida de inserción epitelial comparados con pacientes controles 13. Así mismo, un estudio español encuentra un mayor índice gingival, grado de gingivitis y cálculo entre los pacientes esquizofrénicos que en los pacientes controles (14).

En todos estos estudios, incluido el presente, la prevalencia de destrucción periodontal avanzada (CPITN 4) es muy escasa, sin embargo, el porcentaje y extensión de los sextantes excluidos (CPITN X) puede incrementar los hallazgos de periodontitis grave porque una parte de los dientes perdidos y valorados como sextantes excluidos es debida a razones periodontales. De hecho, esta diferencia era significativa en nuestro estudio.

En los pacientes esquizofrénicos, la salud periodontal empeora con la institucionalización. Thomas et al (12) en 248 pacientes esquizofrénicos crónicos griegos demuestra una peor higiene oral conforme mayor es el tiempo de hospitalización. Estos hallazgos son confirmados por Angelillo et al (15) en Italia, en 193 esquizofrénicos demostrando que la prevalencia de las bolsas periodontales profundas se incrementaba significativamente con el periodo de hospitalización.
Un estudio longitudinal realizado en el Hospital Psiquiátrico de Miraflores de Sevilla ha evaluado la salud periodontal de enfermos esquizofrénicos hospitalizados (11). Los resultados demuestran, significativamente, que durante el periodo de 5 años, ha disminuido el porcentaje de salud periodontal, gingivitis y cálculo, mientras que ha aumentado las bolsas periodontales desde un 36,2\% hasta el 64,8\% (11).

\section{LAS CONDICIONES PERIODONTALES SEGÚN LA EDAD}

Los resultados del presente estudio demuestran que la prevalencia y la extensión de las condiciones periodontales empeora con la edad de los pacientes evaluados, esquizofrénicos y controles. En efecto, cuando se valoran periodontalmente a los enfermos esquizofrénicos, existe un deterioro periodontal. En este sentido, disminuyen la salud periodontal y la gingivitis, mientras que se incrementan las bolsas periodontales y los sextantes excluídos. Una tendencia similar es hallada entre los pacientes sanos controles, donde también se incrementan las bolsas periodontales moderadas y profundas y los sextantes excluidos (Tablas 2 y 6 ).

Nuestros hallazgos en pacientes esquizofrénicos son confirmados por otros estudios nacionales e internacionales $(5,11,15-16)$. Hede (16) en un hospital psiquiátrico de Dinamarca ha demostrado que el número medio de dientes con sangrado gingival, cálculo y bolsas periodontales se incrementaba con la edad de los pacientes hospitalizados. En una población psiquiátrica hospitalizada italiana se encontró un incremento de las bolsas periodontales profundas en relación a la edad, desde el $26,1 \%$ entre los pacientes más jóvenes (menores de 34 años) hasta el $90 \%$ en los pacientes mayores de 75 años (15).

Los estudios sobre la población general confirman esta tendencia ascendente de los trastornos periodontales con la edad (17-18). El estudio nacional sobre la salud bucodental de España demuestra que mientras las bolsas profundas afectaban al 10,7\% de los sujetos de 35-44 años, se incrementaban hasta el $17 \%$ de los sujetos de 65-74 años (17). Otro estudio nacional realizado en Estados Unidos encontraba que la prevalencia de pérdida de inserción moderada y avanzada se incrementa desde el $28 \%$ y $6,4 \%$ respectivamente, en los sujetos de 25-34 años, pasando por el $63 \%$ y $27 \%$ en los de edades comprendidas entre $45-54$ años, hasta alcanzar el $81,5 \%$ y $41,2 \%$ en los mayores de 65 años (18).

La progresión de la patología periodontal con la edad puede ser interpretada también como el aumento de los sextantes excluídos que indica que los dientes 
más afectados periodontalmente se han perdido o su indicación terapéutica es la exodoncia $(11,19)$. Estos hallazgos son confirmados en el presente estudio, dónde la tendencia creciente de los sextantes X está asociada, significativamente, con la edad.

\section{LAS CONDICIONES PERIODONTALES SEGÚN EL SEXO}

En el presente trabajo, aunque las diferencias de prevalencia y extensión de las condiciones periodontales entre sexos, no eran significativas; sin embargo, el análisis muestra un peor estado periodontal con respecto a la prevalencia, en el sexo masculino tanto en los pacientes esquizofrénicos como en los controles con mayor frecuencia de bolsas periodontales, especialmente profundas. (Tabla 3 ).

La explicación de una mayor afectación periodontal entre los pacientes del sexo masculino, puede ser que los hombres suelen presentar peor higiene, más cálculo y placa que las mujeres (20). De hecho un estudio sueco, realizado durante 20 años (1973-93) revela un mayor nivel de gingivitis en 1993 entre los varones que entre las mujeres (21). Además las mujeres suelen utilizar más los servicios dentales, con los beneficios sobre su salud periodontal de cuidados más periódi$\cos (22)$.

Los niveles de placa entree los pacientes con trastornos psicosociales puede estar relacionado con la variable demográfica sexo (23-24). Un estudio realizado en el Reino Unido demuestra que los niveles de placa eran más bajos entre las mujeres comparados con los hombres, lo que es consistente con una mayor severidad de la enfermedad periodontal entre los hombres (23). Otro estudio finlandés valora la relación entre el estilo de vida y el estado periodontal, mostrando que los hombres tienen una significativa mayor proporción de bolsas periodontales que las mujeres (24).

Sin embargo, las diferencias sexuales de los pacientes esquizofrénicos crónicos hospitalizados, no afectan significativamente la progresión de las enfermedades periodontales, a lo largo del tiempo. De hecho, cuando se analizan los hallazgos periodontales en estos pacientes, durante 5 años, se comprueba un incremento en la prevalencia y la extensión de las bolsas moderadas y profundas en ambos sexos (11).

\section{LAS CONDICIONES PERIODONTALES SEGÚN EL TABACO}

El tabaco constituye un factor de riesgo para las enfermedades periodontales (2). Los fumadores no sólo presentan más placa y cálculo, sino que además pueden existir cambios en la flora periodontopatógena y la respuesta inflamatoria periodontal puede estar alterada en los pacientes fumadores; sin olvidar la acción tóxica del tabaco y de la nicotina sobre los tejidos periodontales (25-28). De hecho, los sujetos fumadores pueden presentar una mayor pérdida periodontal, independientemente de la edad y de los niveles de placa, que los sujetos no fumadores (29).

En el presente estudio, la prevalencia y extensión de las bolsas periodontales moderadas y profundas eran mayores entre los pacientes fumadores (esquizofrénicos y controles) comparados con los no fumadores (Tablas 4 y 8). Resultados similares son encontrados entre los trabajadores sanitarios de un hospital israelí donde los sujetos fumadores presentaban una mayor extensión de afectación periodontal con bolsas moderadas (una media de 2,46 sextantes ) que los no fumadores (una media de 1,71 sextantes) (25).

El tabaco está asociado con una mayor susceptibilidad a la periodontitis. Los trastornos psiquiátricos, como la esquizofrenia están consistentemente relacionados con el hábito de fumar, lo que sugiere que los trastornos mentales al incrementar el tabaco indirectamente afectan a los tejidos periodontales (23).

El tabaco es un hábito nocivo frecuente entre las conductas del estilo de vida actual. Los fumadores y exfumadores tienden a tener un negativo estilo de vida con mayores problemas de destrucción periodontal, explicado principalmente por una peor higiene oral. Es decir, los estilos de vida saludables están relacionados, entre otros factores por la ausencia de tabaco, con una baja prevalencia de bolsas periodontales (24).

\section{CONCLUSIONES}

Las enfermedades periodontales y psiquiátricas afectan a un sector importante de la población general. Los trastornos mentales como la esquizofrenia pueden constituir factores de riesgo para el desarrollo y mantenimiento de las alteraciones periodontales. Otros factores frecuentes entre los pacientes psiquiátricos relacionados con la patogenia de las enfermedades periodontales como es el tabaco deben ser identificados para su control. Son necesarios más estudios que relacionen la salud periodontal y la salud general, especialmente los trastornos psíquicos (ej. ansiedad, estrés, depresión). La investigación en el campo de la periodoncia es esencial para el conocimiento de la historia natural de las enfermedades periodontales con el objetivo de mejorar la atención odontológica y médica de estos pacientes. 


\section{ABSTRACT}

The aim of this study was to assess the periodontal conditions in schizophrenic patients compared with control patients.

Patients and methods. 50 schizophrenic patients attended in the Psychiatric Unit at the Virgen Macarena Universitary Hospital of Seville were compared with 50 control patients (without systemic diseases, and drugs) attended in the School of Dentistry of Seville. Periodontal conditions were assessed according to the WHO criteria (CPITN).

Results and Discusion. Schizophrenic patients showed more significative extended excluded sextants $(1,64$ vs 0,42$)$ and prevalence of shallow pockets ( $25,6 \%$ vs $22,4 \%$ ) while control patients showed more calculus $(69,4 \%$ vs $60,5 \%)$ and deep pockets $(6,1 \%$ vs $4,7 \%$ ). Periodontal conditions showed a increased tendency with age. Males (schizophenics and controls) $(32,4 \%$ and $31,3 \%$; respectively) showed a higher prevalence of periodontal pockets than females (22,2\% and 23,5\%). Also, this study showed a higher prevalence and extension of periodontal pockets among smoker patients (schizophrenics and controls).

Conclusions. Relationship between periodontal and psychiatric disturbances must be constitute a field of epidemiological and clinical research for knowledge of natural history of periodontal diseases.

\section{KEY WORDS}

Periodontal conditions, schizophrenic patients, CPITN, mental disorders, periodontal disease.

\section{CORRESPONDENCIA}

\author{
Prof. Eugenio Velasco Ortega \\ C/ José Laguillo ${ }^{\circ} 18-2^{\circ}-6$ \\ 41003 Sevilla \\ Tfno: 954421805 \\ e-mail: evelasco@us.es
}

\section{BIBLIOGRAFÍA}

1. Petersen PE. The World Oral Health Report 2003: continuous improvement of oral health in the 21 st century the approach of the WHO Global Oral Health Programme. Community Dent Oral Epidemiol 2003; 31 (Suppl. 1): 3-24.
2. Tomar SL, Asma S. Smoking attributable periodontitis in the United States: findings from NHANES III. J Periodontol 2000; 71: 743-51.

3. Ramon T, Grinshpoon A, Zusman SP, Weizman A. Oral health and treatment needs of institutionalised chronic psychiatric patients in Israel. Eur Psychiat 2003; 18: 101-5.

4.Velasco E, Machuca G, Martinez-Sahuquillo A, Ríos V, Lacalle J, Bullón P. Dental health among institutionalized psychiatric patients in Spain. Spec Care Dentist 1997; 17: 203-6.

5.Velasco E, Bullón P. Periodontal status and treatment needs among Spanish hospitalized psychiatric patients. Spec Care Dentist 1999; 19: 254-8.

6. Berry H, Landwerlen J. Cigarette smoker's lip lesion in psychiatric patients. J Am Dent Assoc 1973; 86: 657-62.

7. Monteiro da Silva AM, Newman HN, Oakley DA. Psychosocial factors in inflammatory periodontal disease: a review. J Clin Periodontol 1995; 22: 516-26.

8. Monteiro da Silva AM, Oakley DA, Newman HN, Nohl FS, Lloyd HM. Psychosocial factors and adult onset rapidly progressive periodontitis. J Clin Periodontol 1996; 23: 789-94.

9. World Health Organization. Oral Health Surveys. Basic Methods. Ginebra. 1997.

10. Friedlander AH, Friedlander IK; Velasco E, Casas N, Monsalve L. La atención odontológica del paciente con esquizofrenia. Arch Odontoestomatol 2004; 20: 463-73.

11. Velasco C. La evolución de la enfermedad periodontal en una población sin tratamiento. Un estudio de 5 años. Tesis Doctoral. Facultad de Odontología. Universidad de Sevilla. 2000.

12. Thomas A, Lavrentzou E, Karouzos C, Kontis C. Factors which influence the oral condition of chronic schizophrenia patients. Spec Care Dentist 1996; 16: 84-6.

13. Stiefel D, Truelove E, Menard T, Anderson V, Doyle P, Mandel L. A comparison of the oral health of persons with and without chronic mental illness in community settings. Spec Care Dentist 1990; 10: 6-12.

14. Isarría MP, Bravo M, Gomez G, Argente A, Cutando A. Estado oral en pacientes esquizofrénicos institucionalizados. Arch Odontoestomatol Prev Comun 1995; 1: 385-91.

15. Angelillo IF, Nobile CGA, Pavia M, De Facio P, Puca M, Amati A. Dental health and treatment needs in institutionalised psychiatric patients in Italy. Community Dent Oral Epidemiol 1995; 23: 360-4. 
16. Hede B. Oral health in Danish hospitalised psychiatric patients. Community Dent Oral Epidemiol 1995; 23: 44-8.

17. Noguerol B, Llodra JC, Sicilia A, Follana M. La salud bucodental en España. 1994. Madrid: Avances. 1995.

18. Brown LJ, Brunelle JA, Kigman A. Periodontal status in the United States, 1988-1991 : prevalence, extent and demographic variation. J Dent Res 1996; 75 (Spec Iss): 672-83.

19. Miyazaki H, Pilot T, Leclercq MH, Barmes DE. Profiles of periodontal conditions in adults measured by CPITN. Int Dent J 1991; 41: 74-80.

20. Abdellatif HM, Burt BA. An epidemiologic investigation into the relative importance of age and oral hygiene status as determinants of periodontitis. J Dent Res 1987; 66: 13-8.

21. Hugoson A, Norderyd O, Slotte C, Thorstensson H. Oral hygiene and gingivitis in a Swedish adult population 1973, 1983, and 1993. J Clin Periodontol 1998; 25: 807-12.

22. American Academy of Periodontology. Epidemiology of periodontal disease. J Periodontol 1996; 67: 935-45.

23. Monteiro da Silva AM, Newman HN, Oaklay DA, O'Leary R. Psychosocial factors, dental plaque levels and smo- king in periodontitis patients. J Clin Periodontol 1998; 25: 517-23.

24. Sakki TK, Knuutila MLE, Vimpari SS, Hartikainem MSL. Association of lifestyle with periodontal health. Community Dent Oral Epidemiol 1995; 23: 155-8.

25. Goultschin J, Cohen HDS, Donchin M, Brayer L, Soskolne WA. Association of smoking with periodontal treatment needs. J Periodontol 1990; 61: 364-7.

26. Zambon JJ, Grossi SG, Machtei EE, et al. Cigarette smoking increases the risk for subgingival infection with periodontal pathogens. J Periodontol 1996; 67: 1050-4.

27. Kenney EB, Kraal JH, Saxe SR, Jones J. The effects of cigarette smoke on human polymorhonuclear leukocytes. J Periodontol Res 1977; 12: 227-34.

28. Bergstrom J, Elliason G. Cigarette smoking and alveolar bone height in subjects with high standard of oral hygiene. J Clin Periodontol 1987; 14: 466-9.

29. Axelsson P, Paulander J, Lindhe J. Relationship between smoking and dental status in 35-, 50-, 65-, 75-years-old individuals. J Clin Periodontol 1998; 25: 297-305. 